\title{
Urinary System Neoplasm
}

National Cancer Institute

\section{Source}

National Cancer Institute. Urinary System Neoplasm. NCI Thesaurus. Code C3431.

A benign or malignant, primary or metastatic neoplasm involving the urinary system. 